\title{
Neurocritical Care in Germany: Need for Guidance
}

\author{
Hagen B. Huttner $\cdot$ Stefan Schwab
}

Published online: 25 February 2014

(C) Springer Science+Business Media New York 2014

One of the essential dilemmas in (neuro-)critical care is the low level of evidence for or against certain therapeutic approaches. A specific neurocritical care feature-that may complicate further generation of evidence-is its interdisciplinary patient-centered care, i.e., an overlapping patient collective that in Germany is treated either in neurological, neurosurgical, or interdisciplinarily in anesthesiologicaldirected neuro-intensive care units (NICU). For instance, patients with subarachnoidal hemorrhage $(\mathrm{SAH})$ can be treated by neurologists or neurosurgeons as well as anesthesiologists or other specialities. No wonder that the individual guidelines can differ in their recommendations regarding monitoring and treatment [1-3]. Another aspect for the lack of evidence is that large randomized controlled trials generating the highest levels of evidence are less frequent as in general critical care. For the critical care management of stroke, only decompressive surgery is established as a level 1 evidence today [4] whereas many urging problems like management of basilar artery thrombosis or cerebellar stroke reach only weak levels of evidence. Besides practical problems such as randomizing severely ill patients who cannot consent, there is also a lack of industry-sponsored trials in the field of (neuro) critical care, possibly as the severity of diseases undermines the chances for positive clinical end-points [5]. Moreover, high-quality papers funded by public authorities are rare and rather help to guide when there is uncertainty in

This is an editorial for the article available at doi:10.1007/s12028013-9893-3

H. B. Huttner $(\bowtie) \cdot$ S. Schwab

Department of Neurology, University of Erlangen-Nuremberg, Erlangen, Germany

e-mail: hagen.huttner@uk-erlangen.de specific therapeutic options such as whether or not surgery should be performed in ICH or malignant middle cerebral infarction [6-8]. Yet, the majority of basic treatment in daily neurocritical care is supported by lower levels of evidence, if any at all, and studies are often of retrospective and single-center design.

A promising model of bridging the gap between level C and level A evidence is to assimilate and standardize data quality of large tertiary hospitals and to pool data on various treatment aspects. However, prior to designing any future (and even trans-national) collaborations, there is need for an as-built analysis of the current situation of routine clinical management of patients requiring neurocritical care. In this issue of NEUROCRITICAL CARE, authors from Germany (as part of the recently established German-wide IGNITE-group, i.e., Initiative of German NeuroIntensive Trial Engagement) conducted a nationwide online survey which included 50 multiple-choice or open questions regarding admission diagnoses, use of standard operating procedures, protocols, adherence to guidelines and scores, modalities of multimodal neuromonitoring, and target values of distinct blood- and cerebrospinal fluid parameters [9]. All German hospitals with either a specialized neurological, neurosurgical, or interdisciplinary anesthesiological-directed neuro-ICU were contacted, and a sufficient large number of responses (more than $50 \%$ from tertiary University Hospitals) were obtained. The most frequent admission diagnoses were ischemic stroke, intracerebral hemorrhage, subarachnoid hemorrhage, tumor, traumatic brain injury, and epi- or subdural hematomas. Not surprisingly, the admission diagnoses were different depending on the speciality running the NICU. However, as soon as focusing on the clinical scores used and documented baseline parameters, there was a notably high variance across the three major disciplines, i.e., 
neurological versus neurosurgical versus anesthesiological led NICUs, as well as when comparing University and community hospitals. The most common scores were the Glasgow Coma scale or the Hunt \& Hess scale for SAH, whereas more modern scores such as WFNS were typically not obtained. It further turned out that internationally established general ICU-scores such as APACHE or SOFA were also insufficiently applied.

While the parameters qualifying for hemodynamic monitoring, as well as the modus of monitoring, were quite similar, there was a striking variance in terms of initiation and manner of multimodal neuro-monitoring (NM). Despite balanced and established NM concepts [10-12], it appears somehow alarming that the core and key competence of neurocritical care, i.e., performing NM, is almost unpredictable and varies highly across the disciplines running NICUs in Germany [9]. Main reasons to initiate NM were mainly specific diseases as SAH and traumatic brain injury as well as general considerations such as presumed rise in intracranial pressure (ICP) or coma. However, the modus of NM was very heterogeneous with sonography and EEG being the most commonly used techniques followed by ICP monitoring (equally done by external ventricular drains and parenchymal probes) and evoked potentials. Only very few NICUs used probes for monitoring tissue temperature, tissue oxygen, or cerebral blood flow. As stated by the authors, these procedures are time- and man-power-consuming and require a high level of expertise, whereas none-invasive monitoring by sonography or EEG can be done with few technical efforts. Furthermore, the low levels of evidence of advanced multimodal NM may further explain its infrequent use across Germany and that only University hospitals with emphasized vascular focus perform such procedures. Interestingly, if multimodal NM was established, more than $50 \%$ of the responders stated to base clinical decisions on obtained parameters whereas roughly one-third initiated NM for research purposes only [9]. Also, quite surprising are data on clinical consequences of the performed NM. Certain tolerated ICP- or cerebral perfusion pressure values or flow velocities upon sonography in the diagnosis of SAH-related vasospasms vary considerably among the clinical disciplines and hospitals running a NICU.

Despite a substantial number of survey-based methodological shortcomings, we think that the study by Kowoll and colleagues reflects the reality of neurocritical care in Germany. The survey provides valuable information and highlights the key aspects that need to be addressed in the future-i.e., homogenize and standardize clinical management. The authors deserve thanks for their diligent work of having strived toward a current analysis of the situation of neurocritical care. Based on these data, in a second step, the various medical societies involved in the management can redirect their efforts to achieve a further harmonization and standardization of procedures regarding scoring and monitoring. As highlighted by Kowoll and colleagues, there is an overall guideline adherence of less than $75 \%$ [9] which impressively reflects the heterogeneous clinical management both across and even within each of the disciplines. This aspect is most likely due to insufficient guidance from standard procedures for daily clinical routine. Efforts should focus on solving this constraint such that a new fundament arises for future research projects and valid multicenter analyses possibly generating better evidence. Taken together, this study shows how heterogeneous patients requiring neurocritical care are handled-despite the high standard of German medical care system and service. Surveys such as performed by Kowoll and colleagues do help getting an image of current standard of care and courses of actions across Western Countries and may represent a basis for future efforts to standardize neurocritical care processes.

Conflict of interest H.B.H. is part of the IGNITE group on behalf of which the study by Kowoll and colleagues has been published.

\section{References}

1. Diringer MN, Bleck TP, Claude Hemphill J 3rd, et al. Critical care management of patients following aneurysmal subarachnoid hemorrhage: recommendations from the Neurocritical Care Society's Multidisciplinary Consensus Conference. Neurocrit Care. 2011;15:211-40.

2. Connolly ES Jr, Rabinstein AA, Carhuapoma JR, et al. Guidelines for the management of aneurysmal subarachnoid hemorrhage: a guideline for healthcare professionals from the American Heart Association/american Stroke Association. Stroke. 2012;43:1711-37.

3. Steiner T, Juvela S, Unterberg A, Jung C, Forsting M, Rinkel G. European Stroke Organization guidelines for the management of intracranial aneurysms and subarachnoid haemorrhage. Cerebrovasc Dis. 2013;35:93-112.

4. Huttner HB, Schwab S. Malignant middle cerebral artery infarction: clinical characteristics, treatment strategies, and future perspectives. Lancet Neurol. 2009;8:949-58.

5. Mayer SA, Brun NC, Begtrup K, et al. Efficacy and safety of recombinant activated factor VII for acute intracerebral hemorrhage. N Engl J Med. 2008;358:2127-37.

6. Mendelow AD, Gregson BA, Fernandes HM, et al. Early surgery versus initial conservative treatment in patients with spontaneous supratentorial intracerebral haematomas in the International Surgical Trial in Intracerebral Haemorrhage (STICH): a randomised trial. Lancet. 2005;365:387-97.

7. Mendelow AD, Gregson BA, Rowan EN, Murray GD, Gholkar A, Mitchell PM. Early surgery versus initial conservative treatment in patients with spontaneous supratentorial lobar intracerebral haematomas (STICH II): a randomised trial. Lancet. 2013;382:397-408.

8. Hofmeijer J, Kappelle LJ, Algra A, Amelink GJ, van Gijn J, van der Worp HB. Surgical decompression for space-occupying cerebral infarction (the hemicraniectomy after middle cerebral artery infarction with life-threatening edema trial [HAMLET]): a multicentre, open, randomised trial. Lancet Neurol. 2009;8:326-33. 
9. Kowoll CM, Dohmen C, Kahmann J, et al. Standards of scoring, monitoring, and parameter targeting in german neurocritical care units: a national survey. Neurocrit Care 2013;1-11. doi:10.1007/ s12028-013-9893-3.

10. Stuart RM, Schmidt M, Kurtz P, et al. Intracranial multimodal monitoring for acute brain injury: a single institution review of current practices. Neurocrit Care. 2010;12:188-98.
11. Hemphill JC, Andrews P, De Georgia M. Multimodal monitoring and neurocritical care bioinformatics. Nat Rev Neurol. 2011;7:451-60.

12. Stocchetti N, Roux PL, Vespa P, et al. Clinical review: neuromonitoring —an update. Crit Care. 2013;17:201. 